\title{
Proximale Femurfrakturen bei liegender Hüftgelenkstotalendoprothese
}

\author{
Richard Stange, Moritz Freistühler, Philipp Michel, Clemens Kösters, Michael J. Raschke
}

\section{Zusammenfassung}

Die Inzidenz periprothetischer Frakturen am proximalen Femur bei liegender Hüftgelenksprothese nimmt aufgrund der demografischen Entwicklung und der damit verbundenen höheren Lebenserwartung, der steigenden Zahl der implantierten Prothesen sowie des vermehrten Auftretens von Osteoporose und des höheren Aktivitätsniveaus der älteren Bevölkerung zu. Neben der Lokalisation der Fraktur und der Stabilität der Prothese sind insbesondere auch patientenspezifische individuelle Faktoren für die Planung des therapeutischen Vorgehens zu berücksichtigen. Für diese Frakturform hat sich in den letzten Jahren die Vancouver-Klassifikation nach Duncan und Masri im klinischen Alltag durchgesetzt. Sie berücksichtigt neben der Lokalisation der Fraktur und der Stabilität der Prothese auch die Knochenqualität und liefert so Hinweise für die Auswahl des richtigen Behandlungsverfahrens. Die Thera- pie der proximalen periprothetischen Femurfraktur ist sehr differenziert und reicht von der konservativen Therapie über die operative Stabilisierung bis hin zum totalen endoprothetischen Femurersatz, und sollte unter optimaler Vorbereitung und besten Operationsbedingungen an spezialisierten Zentren erfolgen. Neue interdisziplinäre multimodale Therapieansätze können helfen, der zu erwartenden weltweiten Zunahme der periprothetischen Frakturen zu begegnen.

\section{Proximal Femoral Fractures around a Total Hip Arthroplasty}

The incidence of periprosthetic fractures around a total hip arthroplasty is increasing with the demographic development involving the increased expectation of life, the increased number of endoprostheses and the increased incidence of osteoporosis in combination with higher levels of activity in the elderly population. In addition to the localisation of the fracture and the stability of the prosthesis, especially patient-specific aspects must be taken into account when planning the therapeutic procedure. The Vancouver classification developed by Duncan and Masri has prevailed for this type of fracture in the past years in clinical practice. It considers not only the location of the fracture and the stability of the prosthesis but also the bone quality and thus provides hints for the selection of the right therapeutic procedure. The therapy for the periprosthetic proximal femoral fracture is very differentiated ranging from conservative treatment over surgical stabilisation to total femoral replacement and should therefore be carried out under optimum preparation and operation conditions at specialised centres. New interdisciplinary multimodal treatment approaches will help us to manage the expected global increase of periprosthetic fractures.

\section{Epidemiologie}

Die Inzidenz periprothetischer Frakturen des Hüftgelenks nimmt aufgrund der demografischen Entwicklung, höherer Lebenserwartung und der steigenden Zahl der implantierten Prothesen zu. In Europa nimmt Deutschland bei der Anzahl an implantierten Hüftendoprothesen mit ca. 254000 im Jahr 2008 eine Spitzenposition ein. Die Revisionsrate lag hier bei $15,1 \%$ und es wurden insgesamt ca. 45000 Prothesenwechseloperationen durchgeführt [6]. In der Literatur variieren die Inzidenzangaben für periprothetische proximale Femurfrak-

OP-JOURNAL 2015; 31: 22-27

(c) Georg Thieme Verlag KG Stuttgart · New York DOI http://dx.doi.org/10.1055/s-0035-1545872 turen zwischen $0,045 \%$ und mehr als 27,8\% [2,3,16,19], wobei die Anzahl nach primärer Endoprothesenimplantation geringer ist. Die Inzidenz der periprothetischen proximalen Femurfraktur liegt nach Meek et al. 5 Jahre nach Implantation einer primären Totalendoprothese bei $0,9 \%$ und $4,2 \%$ bei Revisionsprothesen [19]. Beals und Tower konnten in einer weiteren Arbeit zeigen, dass ein höheres Risiko, eine periprothetische Fraktur zu erleiden, nach zementfreier gegenüber der zementierten Schaftimplantation insbesondere in den ersten 6 Monaten vorliegt [1]. In einer großen Registerstudie konnten Lindahl et al. zeigen, dass die durchschnittliche Standzeit bis zur Fraktur für die primäre Endoprothese bei 7,4 Jahren liegt. Bei der Revisionsprothese verringert sich diese Zeit auf 3,9 Jahre und wird mit der steigenden
Anzahl an Revisionsoperationen immer kürzer (3,8 Jahre nach der 2. Revision und 2,3 Jahre nach der 3. Revision) [16].

\section{Risikofaktoren}

Bei den Risikofaktoren der periprothetischen Fraktur lassen sich allgemeine von lokalen Faktoren unterscheiden.

Zu den allgemeinen Risikofaktoren zählen systemische Nebenerkrankungen, die die Knochenqualität beeinflussen. Hier ist in erster Linie die Osteoporose als einer der häufigsten Risikofaktoren insbesondere bei Frauen im postmenopausalen Alter zu nennen.

Des Weiteren führt die Abnahme der Koordinationsfähigkeit, Muskulatur und Sinneswahrnehmung in Kombination 


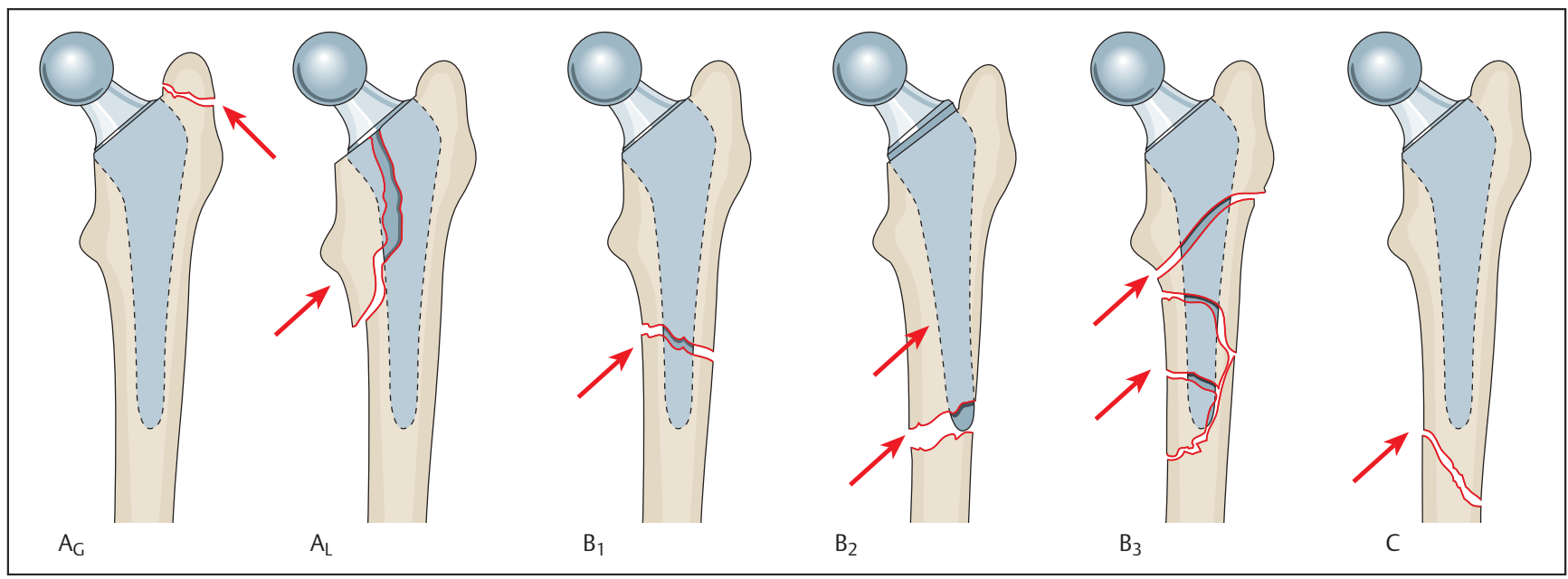

Abb. 1 Schematische Darstellung der Vancouver-Klassifikation nach Duncan und Masri [4].

Tab. 1 Übersichtsdarstellung des United Classification Systems, einer Klassifizierung unabhängig von der Lokalisation der Prothese nach Duncan und Haddad [5].

\begin{tabular}{ll} 
Typ & Charakteristika \\
\hline A & Apophyse/Tuberositas mit Ansatz von Weichteilgewebe \\
\hline B & Fraktur im Bereich des Implantatbetts \\
\hline C & Fraktur distal oder proximal des Implantatbetts \\
\hline D & Knochen mit 2 Gelenkprothesen betroffen \\
\hline E & 2 Knochen um eine Prothese betroffen \\
\hline F & Gelenkfläche, die in Verbindung mit einer Prothese steht, ist betroffen
\end{tabular}

mit einer zunehmenden Morbidität des alten Menschen $\mathrm{zu}$ rezidivierenden Sturzereignissen. Diese Stürze sind als Niedrigenergietrauma i.d.R. ursächlich für eine periprothetische Fraktur. $\mathrm{Zu}$ sätzlich steigt beim älteren Menschen das Risiko, eine Fraktur zu erleiden mit der Anzahl der eingenommenen Medikamente [13]. Weitere allgemeine Risikofaktoren stellen die Gruppe der neurodegenerativen Erkrankungen (z.B. Morbus Parkinson) und die rheumatoide Arthritis dar.

Zu den lokalen Risikofaktoren zählt insbesondere die Schaftlockerung, die durch Reduktion der Steifigkeit und des Drehmoments zu Frakturen führt [9] (Abb.4).

Die Implantationstechnik kann einerseits durch nicht ausreichendes Press Fit bei der zementfreien Technik und andererseits durch zu starkes Aufbohren oder Aufraspeln im Verlauf zu Frakturen führen. Die Revisionsendoprothetik besitzt aufgrund von Osteolysen, verminderter Knochenqualität und -quantität sowie schwieriger Zement- und Implantatent- fernungen mit ggf. notwendiger Osteotomie eine höhere Inzidenz an periprothetischen Frakturen.

\section{Klassifikationen}

In den letzten Jahren hat sich im klinischen Alltag bei den periprothetischen Frakturen am proximalen Femur die sog. Vancouver-Klassifikation nach Duncan und Masri durchgesetzt [4] (Abb. 1). Hier werden mit der anatomischen Lokalisation, der Prothesenstabilität und der Knochenqualität 3 wesentliche Aspekte für eine therapeutische Intervention berücksichtigt. Proximale Frakturen im Bereich der Trochanterregion werden hier als Typ-A-Fraktur bezeichnet, Brüche entlang des Prothesenschafts werden dem Typ B und Frakturen unterhalb der Prothesenspitze dem Typ C zugeordnet. Frakturen des Typs B werden hier noch weiter unterteilt, je nach Vorliegen einer stabilen Prothese (Typ B1), lockeren Prothese (Typ B2) oder lockeren Prothese bei gleichzeitig schlechter Knochenqualität (Typ B3). Ein Versuch, eine einheitliche Klassifikation für alle Arten der periprothetischen Fraktur zu erstellen, ist das
Unified Classification System (UCS) von Duncan und Haddad [5] (Tab. 1). Hier wird in Typen von A-F jede Möglichkeit des Bruches um eine oder mehrere Prothesen sowie in einem oder beiden gelenkbildenden Knochen beschrieben.

\section{Therapieplanung und Vorbereitung}

Hier muss zwischen intraoperativ aufgetretenen Frakturen, die sofort versorgt werden müssen, und postoperativen Frakturen unterschieden werden.

Die Auswahl des richtigen Behandlungsverfahrens richtet sich nach Lokalisation der Fraktur, möglichem Dislokationsgrad, Festigkeit der Prothese und der Knochenqualität, also den Faktoren, die idealerweise in einer Klassifikation mit abgebildet werden [26].

Zusätzlich müssen weitere Faktoren wie Anspruch und biologisches Alter des Patienten und mögliche Nebenerkrankungen berücksichtigt werden. Auch die Anzahl der Voroperationen, die mögliche zusätzliche endoprothetische Versorgung angrenzender Gelenke sowie auch die Erfahrung des Operateurs müssen in dem Entscheidungsprozess berücksichtigt werden. Aufgrund dieser sehr komplexen Situationslage und des zusätzlich meist multimorbiden Patientenguts sollte die Versorgung dieser Frakturen an speziellen Zentren durchgeführt werden. Eine sofortige Notfalloperation der periprothetischen Fraktur ist bei offenen Frakturen, großem Weichteilschaden oder Gefäß- oder Nervenschädigungen erforderlich. Meist ist jedoch neben einer suffizienten Analgesie eine Ruhigstellung in einer Schiene oder die Anlage einer Extension ausreichend, um die 
mehrheitlich multimorbiden Patienten interdisziplinär optimal auf die Operation vorzubereiten und den Eingriff in den ersten Tagen nach optimaler Vorbereitung sowie unter optimalen Operationsbedingungen durchführen zu können. Ziel der operativen Versorgung ist eine möglichst frühfunktionelle Nachbehandlung unter möglichst belastungsstabilen Verhältnissen.

\section{Behandlungsalgorithmus}

Das eigene klinische Vorgehen soll hier an einem Algorithmus anhand der Vancouver-Klassifikation erläutert werden. Hierbei ist neben der Frakturlokalisation und Morphologie, die sich im Röntgenbild meist gut darstellen lässt, die Frage der Stabilität der Prothese von entscheidender Bedeutung. Hier muss auf radiologische Lockerungszeichen wie Saumbildung um den Prothesenschaft, Osteolysen, Frakturen im Zementmantel und mögliche Zeichen für Verschleiß geachtet werden (Dezentrierung des Kopfes in der Pfanne etc.). Ein Vergleich mit Voraufnahmen, wenn vorhanden, sollte immer durchgeführt werden. Zusätzlich kann auch die Anamnese über belastungsabhängige Schmerzen, Gangunsicherheiten, Stauchungsschmerz oder Infekt in den Wochen vor der Fraktur Hinweise auf eine mögliche Lockerung liefern. Zum Ausschluss einer Lockerung kann die Durchführung einer präoperativen Computertomografie hilfreich sein. Bestehen Zweifel an der Festigkeit der Prothese, muss diese intraoperativ auf Stabilität überprüft werden und im Zweifel die Prothese gewechselt werden, um Folgeoperationen zu vermeiden.

\section{Typ-A-Frakturen}

Dieser Frakturtyp macht nach Lindahl et al. [17] ca. 2-3\% aller periprothetischen hüftgelenksnahen Frakturen aus und ist meist stabil. Es wird die Fraktur des Trochanter major (Typ $A_{G}$ ) von der des Trochanter minor (Typ $A_{L}$ ) unterschieden. Die postoperativ aufgetretene Fraktur des Trochanter major ist eine Domäne der konservativen Therapie [10,21]. Hier sollten für 6-12 Wochen forcierte Abduktionsbewegungen vermieden und, wenn möglich, eine Teilbelastung der betroffenen Extremität durchgeführt werden. Bei persistierenden Schmerzen, Trendelenburg-Hinken, Instabilitätsgefühl nach Pseudarthrosenausbildung oder rezidivierenden Luxationen ist jedoch eine Indikation zur operativen Refixierung gegeben. Ebenfalls ist bei einer
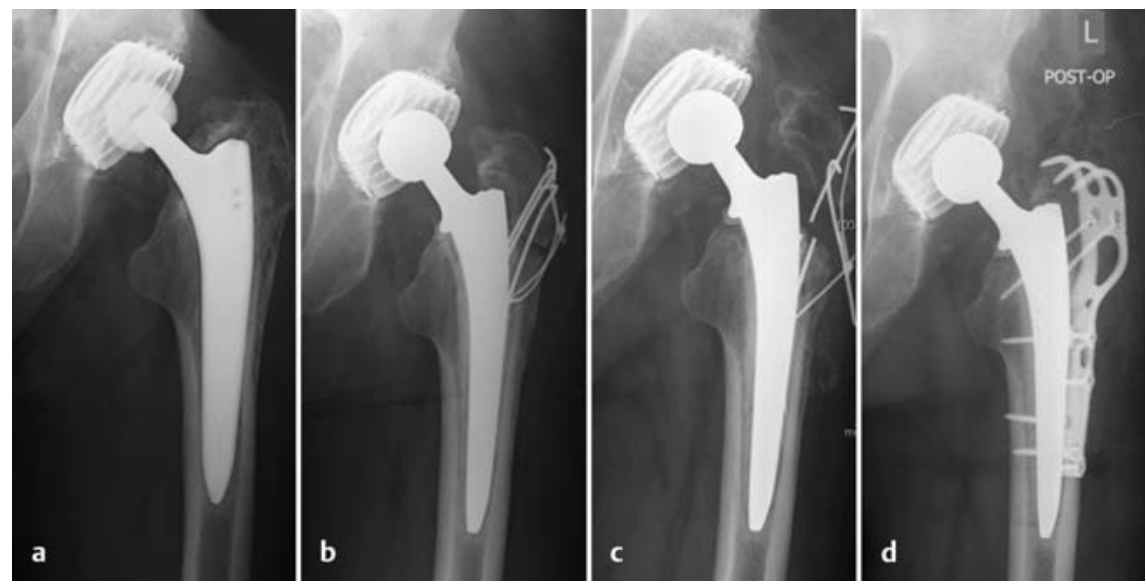

Abb. $\mathbf{2}$ a bis d a 75-jähriger Patient mit Schaftlockerung; b Prothesenwechsel inkl. Trochanterosteotomie und Refixierung des Trochanter major mittels Drahtcerclage; c 7 Monate später Vorstellung mit persistierenden Beschwerden und Trendelenburg-Hinken. d Postoperatives Röntgenbild nach Entfernung der Cerclagen und Refixierung des Trochanter major mittels Trochanterplatte (Trofix, Fa. Zimmer).

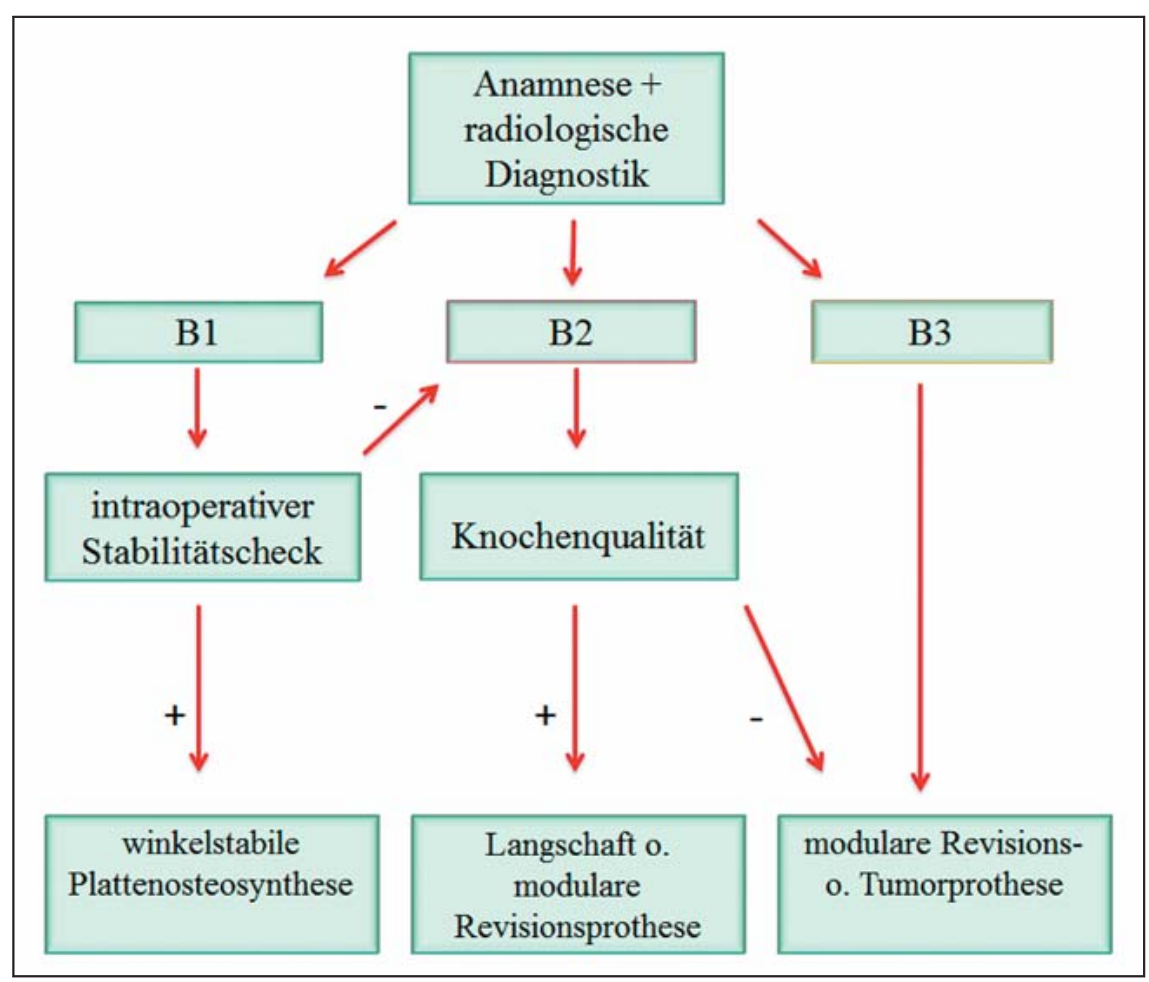

Abb. 3 Algorithmus für das therapeutische Vorgehen bei Vancouver-B-Frakturen.

Dislokation der Fragmente um mehr als $2 \mathrm{~cm}$ die operative Therapie der konservativen überlegen [21]. Eine intraoperative Fraktur des Trochanter major sollte immer sofort fixiert werden. Ist die Entscheidung zur operativen Therapie gefallen, stehen verschiedene CerclageTechniken, Plattensysteme und Kombinationen zur Auswahl. Die isolierte periprothetische Fraktur des Trochanter minor ist hingegen selten und wird in nahezu allen Fällen konservativ behandelt (Abb. 2).

\section{Typ-B-Fraktur}

Die Typ-B-Fraktur der Vancouver-Klassifikation stellt mit einer Inzidenz von $88 \%$ die mit Abstand häufigste Frakturform dar. Hiervon macht die B2-Fraktur mit einem Bruch im Bereich des Prothesenschafts und gelockerter Prothese wiederum über die Hälfte aller Fälle aus und ist somit die häufigste periprothetische Fraktur am Hüftgelenk [17]. Essenziell für die Wahl des richtigen Therapieverfahrens ist hier die Stabilität der einlie- 


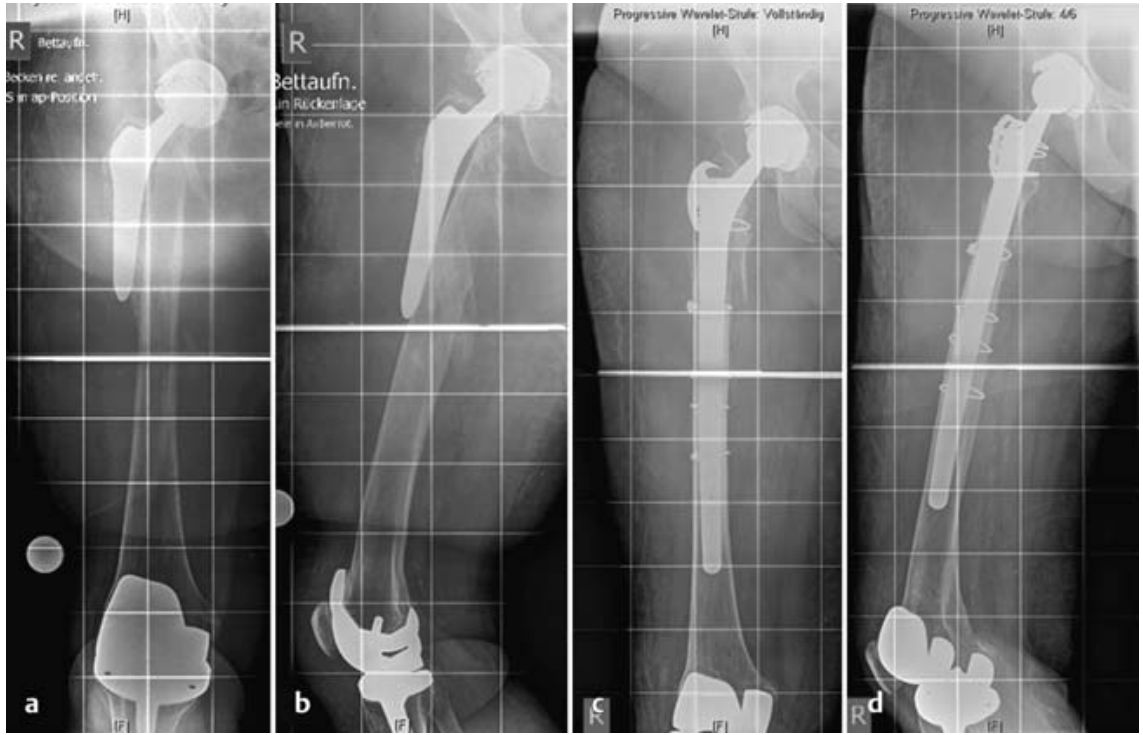

Abb. 4a bis d Vancouver-Typ-B2-Fraktur bei einer 75-jährigen Patientin nach Sturz in häuslicher Umgebung auf die rechte Hüfte $(\mathbf{a}, \mathbf{b})$. Postoperative Bilder nach Prothesenwechsel auf modulare Revisionsendoprothese (Revitan, Fa. Zimmer) inkl. Cerclagen und Trochanter-Reattachment Device (Fa. DePuy Synthes; c, d).

genden Prothese. Nach Anamnese, klinischer Untersuchung sowie radiologischer Diagnostik kann präoperativ eine Einteilung der Vancouver-B-Fraktur in ihre Subtypen und die Operationsplanung erfolgen (Algorithmus Abb. 3).

Es muss intraoperativ zwingend eine Kontrolle und Reevaluation der Prothesenstabilität erfolgen, um Folgeoperationen zu vermeiden. Vermeintliche B1Frakturen können sich intraoperativ als B2-Frakturen mit gelockerter Prothese herausstellen, deren Lockerung in der präoperativen Diagnostik nicht nachweisbar war.

Der Operateur muss in der Lage sein, das gewählte Therapieverfahren ggf. auch intraoperativ von einem Osteosyntheseverfahren auf einen Prothesenwechsel und umgekehrt umstellen zu können. Dies erfordert einen hohen Grad an Expertise in der Osteosynthese und in der Endoprothetik sowohl des Operateurs als auch des gesamten Operationsteams und eine entsprechende Vorhaltung der speziellen Implantate und Prothesen, wie es zumeist nur an speziellen Zentren zu finden ist.

Die Therapie der B1-Fraktur und somit der stabilen Hüftprothese sollte unter der Verwendung von Plattenosteosyntheseverfahren erfolgen. Durch die höhere biomechanische Stabilität ist bei winkelstabilen Plattensystemen zumeist die sofortige Mobilisation mit Teilbelastung möglich. Hier konnten in der Litera- tur gute Heilungsraten von bis zu $91 \%$ erreicht werden [8]. Entscheidend für die Osteosynthese ist, dass das distale Prothesenende um mindestens 2 Femurschaftbreiten überbrückt wird [24]. Sowohl proximal als auch distal der Fraktur sollten für eine stabile Versorgung 8 Kortikales gefasst werden. Hierbei ist auch auf eine ausreichende Länge der Platten zu achten und auf einen ausreichenden Abstand der Schrauben vom Frakturspalt, damit die Osteosynthese nicht zu rigide wird und es zur Ausbildung von Pseudarthrosen kommt. Heute gibt es moderne winkelstabile Plattensysteme, welche speziell für die periprothetische Fraktur entwickelt wurden und sich u.a. durch polyaxiale Winkelstabilität auszeichnen [22,26]. Dies ermöglicht das Einbringen und Platzieren von Schrauben am Schaft der Prothese vorbei bikortikal im Knochen. Studien konnten deutliche biomechanische Vorteile dieser bikortikalen Schraubenfixierung der Platte gegenüber einer Fixierung mit monokortikalen Schrauben oder Cerclagen nachweisen [14]. Ein Beispiel für diese speziellen Plattensysteme ist die „Non-Contact Bridging Plate“ bzw. „Non-Contact Periprosthetic Plate“ der Firma Zimmer (Warsaw, IN, USA). Hier kann der Operateur, je nach Lokalisation am Femur, zwischen mehreren anatomisch präformierten Platten unterschiedlicher Länge wählen. Es besteht eine diagonale Dreilochanordnung und es ist eine polyaxiale Winkelstabilität mit einer Angulation von maximal $30^{\circ}$ möglich. Eine Alternative ist die „Variable Angle Locking Com- pression Plate" der Firma DePuy Synthes (Solothurn, Schweiz). Diese bietet in Kombination mit der „Locking Attachment Plate" und weiteren Kombinationsplatten ebenfalls die Möglichkeit der polyaxialen Winkelstabilität an jeder Lokalisation des Femurs. Diese Implantate können dann teils auch minimalinvasiv über Zielbügel eingeschoben werden und ermöglichen somit eine weichteilschonende Operationstechnik. Des Weiteren ist eine zusätzliche Kombinationsmöglichkeit der Plattenosteosynthese mit Cerclagesystemen zur zusätzlichen Fragmentfixierung möglich. Neben der herkömmlichen Drahtcerclage hat sich in den letzten Jahren zunehmend die Kabelcerclage aufgrund einer standardisierten und einfachen Anlage sowie der hohen Versagenslast etabliert [25]. Lenz et al. konnten hier in einer biomechanischen Testreihe zeigen, dass die Kombination aus Kabelcerclage und einer doppelt um den Knochen gelegten Schlinge die stabilste Fragmentfixierung aufwies [15]. Bei zementierten Schäften sollten spezielle größere Zementbohrer zur Vermeidung von Rissen im Zementmantel benutzt werden [11]. Bei Patienten mit Vancouver-B1- oder -C-Fraktur und zusätzlich schlechter Knochenqualität oder Defekten bei Osteoporose ist ggf. zur Gewährleistung einer belastungsoder übungsstabilen Situation eine Doppelplattenosteosynthese mit einer zusätzlichen medialen oder ventralen Platte indiziert [22].

Bei den Vancouver-Typ-B2-Frakturen ist ein Prothesenwechsel auf einen diaphysär verankernden Schaft indiziert.

Um die Prothese stabil zu verankern, sollte die Fraktur um mindestens $2 \mathrm{Fe}-$ murschaftbreiten (mindestens $7-10 \mathrm{~cm}$ ) von der Prothesenspitze überragt werden (Abb. 4). Eine zu lange Prothese sollte jedoch nicht gewählt werden, da das kürzeste stabile Implantat die besten Langzeitergebnisse liefert $[10,23]$. Eine Alternative sind proximal verankernde Schäfte mit distaler Verriegelung. Diese Verriegelung wird nach ca. 6-9 Monaten entfernt und ermöglicht ein nachträgliches Einsintern der Prothese nach Frakturheilung und somit eine proximale Verankerung. Ein Nachteil ist jedoch die zusätzliche Schwächung des Knochens durch das Bohrloch mit der Gefahr einer zusätzlichen Fraktur [10]. Die Reposition und Fragmentsicherung an den Prothesenschaft kann durch Cerclagen erfolgen. Ein Prothesenwechsel ist prinzipiell zementiert und zementfrei möglich. Der 


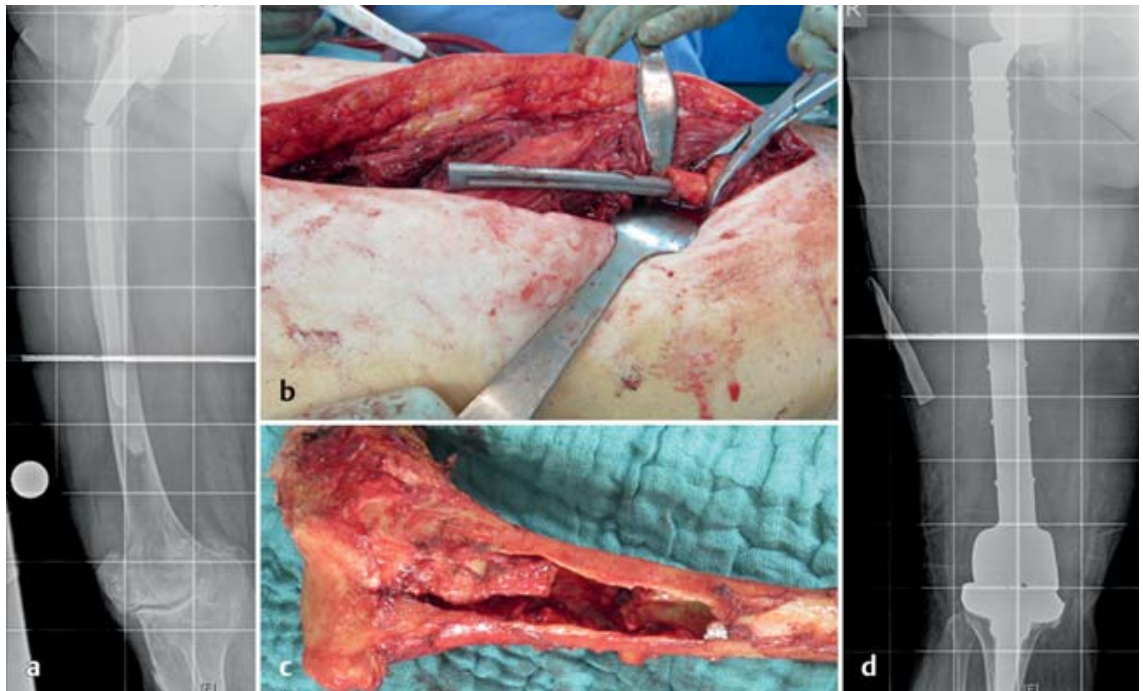

Abb.5a bis d Vancouver-B3-Fraktur mit Prothesenbruch einer zementierten Langschaftprothese. Im Bereich des proximalen Femurs ist eine deutliche Saumbildung als Zeichen für eine Lockerung zu erkennen. Ebenfalls besteht bereits fortgeschrittene Gonarthrose (a). Intraoperativ deutlich verminderte Knochenqualität $(\mathbf{b}, \mathbf{c})$. Postoperatives Bild des totalen Femurersatzes (MUTARS Fa. Implantcast; d).
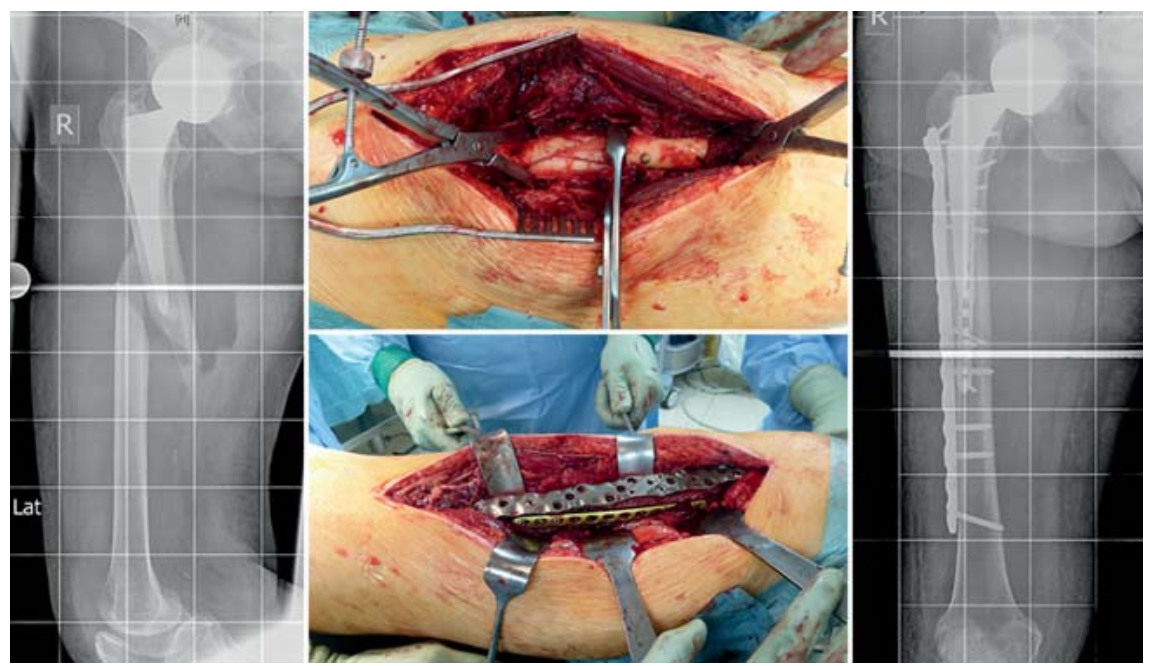

Abb. 6 Fraktur Vancouver Typ C mit stabiler Prothese. Zunächst anatomische Reposition und Osteosynthese mittels freier 3,5-mm-Schraube dann Doppelplattenosteosynthese mittels 12Loch-NCP-PP (Fa. Zimmer) und 12-Loch-3,5-mm-LCP (Fa. DePuy Synthes).

Nachteil des zementierten Wechsels bei der periprothetischen Fraktur ist die Möglichkeit der Ausbildung von Pseudarthrosen, da der Zement in die Frakturspalten eindringen kann und so eine suffiziente Knochenheilung erschwert. Deshalb ist ein zementierter Wechsel nur bei alten Patienten mit deutlich verminderter Knochenqualität zu empfehlen [24]. Prinzipiell lassen sich mit der modularen Revisionsprothese und der nicht modularen Langschaftprothese 2 Formen der Revisionsprothese für die periprothetische Fraktur unterscheiden. Bei den modularen Prothesen ergibt sich der Vorteil, dass durch die schrittweise Im- plantation der Prothese eine Anpassung an die individuellen und frakturbedingten Gegebenheiten möglich ist. Schwachpunkte dieser Prothesen sind jedoch die Verbindungsstellen zwischen den Komponenten, die in einigen Arbeiten zu einem sog. „fretting“, einem vermehrten Schwingreibungsverschleiß und so $\mathrm{zu}$ Osteolysen geführt hat $[10,20,27]$.

Die Typ-B3-Fraktur ist durch eine gelockerte Prothese mit zusätzlich verminderter Knochenqualität oder Verlust an Knochenmasse gekennzeichnet. Hier kommen dann zumeist modulare Revisions- oder Tumorprothesen bis hin zum kompletten Ersatz des Femurs zum Einsatz (Abb.5). Um eine stabile Verankerung zu gewährleisten, gelten die gleichen Prinzipien wie für den bereits zuvor beschriebenen Prothesenwechsel auf diaphysär verankernde Schäfte bei B2-Frakturen. Aufgrund des Vorteils der sofortigen Belastungsstabilität wird jedoch bei schlechter Knochenqualität ein zementierter Ersatz des proximalen Femurs und je nach Situation sogar des gesamten Femurs von einigen Autoren empfohlen $[10,12,18]$. Beim Ersatz des proximalen Femurs kommt es zum Verlust der Muskelansätze, die durch spezielle Anbindungsschläuche an die Prothese fixiert werden müssen. Zusätzlich kommen als Luxationsschutz größere Köpfe und überhöhte Inlays oder Pfannen zum Einsatz. McLean et al. konnten hier in einer Arbeit zeigen, dass der Ersatz des proximalen und sogar des totalen Femurs zu guten klinischen Ergebnissen nach periprothetischer Fraktur und schlechter Knochenqualität führen kann [18].

\section{Typ-C-Fraktur}

Dieser Frakturtyp findet sich außerhalb des Verankerungsbereichs der Prothese und wird wie die B1-Fraktur mittels Plattenosteosynthese versorgt.

Es gelten hier die gleichen Versorgungsprinzipien für die Plattenfixierung im Bereich des Prothesenschafts wie für die B1-Fraktur. Es sollte ebenfalls eine möglichst belastungsstabile Situation ggf. auch mittels Doppelplattenosteosynthese angestrebt werden (s. Abb. 6). Zur besseren Verankerung der Schrauben kann insbesondere am distalen Femur bei osteoporotischem Knochen ggf. eine Augmentierung des Implantatlagers erfolgen. Dies hat eine Erhöhung der Stabilität durch Vergrößerung des Knochen-Implantat-Interfaces durch Ummantelung mit Knochenzement zur Folge [26]. In seltenen Fällen ist eine Osteosynthese auch mit einem retrograden Verriegelungsnagel indiziert.

\section{Prävention}

Um der zu erwartenden weltweiten Zunahme der periprothetischen Fraktur zu begegnen, sollte in den nächsten Jahren der Prävention ein zunehmender Stellenwert eingeräumt werden.

Der Ansatz sollte hier interdisziplinär und multimodal sein. Es gilt zum einen, beim alten Menschen Sturzereignisse zu 
verhindern, indem begünstigende Komorbiditäten wie z.B. neurodegenerative, ophthalmologische oder kardiovaskuläre Erkrankungen möglichst optimal therapiert und eingestellt werden.

Die Osteoporose als ein Hauptfaktor für die schlechte Knochenqualität und damit für Frakturen muss leitliniengerecht therapiert werden.

Des Weiteren gilt es, durch regelmäßige Nachsorgeuntersuchungen der Patienten eine schlechte Knochenqualität und Lockerungen von Endoprothesen zu detektieren, bevor es zum Frakturereignis kommt. Studien konnten zeigen, dass auch eine interdisziplinäre Betreuung der meist geriatrischen Patienten nach erlittener Fraktur zu einem besseren Outcome und kürzeren stationären Aufenthalten führt [7].

\section{Schlussfolgerungen}

- Die Inzidenz periprothetischer Frakturen des Hüftgelenks nimmt aufgrund der demografischen Entwicklung, höherer Lebenserwartung und der steigenden Zahl der implantierten Prothesen zu.

- Die Osteoporose als ein Hauptfaktor für die schlechte Knochenqualität, geriatrische Begleiterkrankungen und häufig mehrfache endoprothetische Versorgung führen beim alten Patienten zu einem erhöhten Risiko für periprothetische Frakturen und erfordern ein komplexes Management.

- Bei den Risikofaktoren für eine periprothetische Fraktur lassen sich allgemeine von lokalen Faktoren unterscheiden.

- Die Auswahl des richtigen Behandlungsverfahrens richtet sich nach Klassifikation und Lokalisation der Fraktur, möglichem Dislokationsgrad, Festigkeit der Prothese und der Knochenqualität.

- Unabdingbar ist eine regelmäßige Kontrolle der Patienten mit Prothese, um eine Lockerung der Implantate, geminderte Knochenqualität oder Sturzneigung frühzeitig zu erkennen und zu behandeln, bevor es zum Frakturereignis kommt.

\section{Literatur}

${ }^{1}$ Beals RK, Tower SS. Periprosthetic fractures of the femur. An analysis of 93 fractures. Clin Orthop Relat Res 1996; 327: 238-246

2 Berry DJ. Epidemiology: hip and knee. Orthop Clin North Am 1999; 30: 183-190

3 Della Rocca GJ, Leung KS, Pape HC. Periprosthetic fractures: epidemiology and future projections. J Orthop Trauma 2011; 25 (Suppl. 2): S66-S70

4 Duncan CP, Masri BA. Fractures of the femur after hip replacement. Instr Course Lect 1995; 44: 293-304

${ }^{5}$ Duncan CP, Haddad FS. The Unified Classification System (UCS): improving our understanding of periprosthetic fractures. Bone Joint J 2014; 96-B: 713-716

${ }^{6}$ Falbrede I, Widmer M, Kurtz $S$ et al. [Utilization rates of lower extremity prostheses in Germany and Switzerland: a comparison of the years 2005-2008]. Orthopade 2011; 40: 793-801

${ }^{7}$ Friedman SM, Mendelson DA, Bingham KW et al. Impact of a comanaged Geriatric Fracture Center on short-term hip fracture outcomes. Arch Intern Med 2009; 169: 1712-1717

${ }^{8}$ Graham SM, Moazen M, Leonidou A et al. Locking plate fixation for Vancouver B1 periprosthetic femoral fractures: a critical analysis of 135 cases. J Orthop Sci 2013; 18: 426436

${ }^{9}$ Harris B, Owen JR, Wayne JS et al. Does femoral component loosening predispose to femoral fracture?: an in vitro comparison of cemented hips. Clin Orthop Relat Res 2010; 468: 497-503

${ }^{10}$ Holzapfel BM, Prodinger PM, Hoberg $M$ et al. [Periprosthetic fractures after total hip arthroplasty: classification, diagnosis and therapy strategies]. Orthopade 2010; 39: 519535

11 Kampshoff J, Stoffel KK, Yates PJ et al. The treatment of periprosthetic fractures with locking plates: effect of drill and screw type on cement mantles: a biomechanical analysis. Arch Orthop Trauma Surg 2010; 130: 627-632

12 Klein GR, Parvizi J, Rapuri V et al. Proximal femoral replacement for the treatment of periprosthetic fractures. J Bone Joint Surg Am 2005; 87: 1777-1781

${ }^{13}$ Lai SW, Liao KF, Liao CC et al. Polypharmacy correlates with increased risk for hip fracture in the elderly: a population-based study. Medicine (Baltimore) 2010; 89: 295-299

${ }^{14}$ Lenz M, Perren SM, Gueorguiev B et al. Mechanical behavior of fixation components for periprosthetic fracture surgery. Clin Biomech (Bristol, Avon) 2013; 28: 988-993

15 Lenz M, Perren SM, Richards RG et al. Biomechanical performance of different cable and wire cerclage configurations. Int Orthop 2013; 37: 125-130

${ }^{16}$ Lindahl $H$, Malchau $H$, Herberts $P$ et al. Periprosthetic femoral fractures classification and demographics of 1049 periprosthetic femoral fractures from the Swedish National
Hip Arthroplasty Register. J Arthroplasty 2005; 20: 857-865

17 Lindahl H, Garellick G, Regner $H$ et al. Three hundred and twenty-one periprosthetic femoral fractures. J Bone Joint Surg Am 2006; 88: 1215-1222

${ }^{18}$ McLean AL, Patton JT, Moran M. Femoral replacement for salvage of periprosthetic fracture around a total hip replacement. Injury 2012; 43: 1166-1169

${ }^{19}$ Meek RM, Norwood T, Smith R et al. The risk of peri-prosthetic fracture after primary and revision total hip and knee replacement. J Bone Joint Surg Br 2011; 93: 96-101

20 Molloy DO, Munir S, Jack CM et al. Fretting and corrosion in modular-neck total hip arthroplasty femoral stems. J Bone Joint Surg Am 2014; 96: 488-493

${ }^{21}$ Pritchett JW. Fracture of the greater trochanter after hip replacement. Clin Orthop Relat Res 2001; 390: 221-226

22 Raschke MJ, Stange R, Kosters C. [Treatment of periprosthetic and peri-implant fractures: modern plate osteosynthesis procedures]. Unfallchirurg 2012; 115: 1009-1021

${ }^{23}$ Schuh A, Holzwarth U, Zeiler G. Titanium modular revision prosthesis stem in revision hip prosthesis. Orthopade 2004; 33: 63-67

24 Stange R, Raschke MJ, Fuchs T. [Periprosthetic fractures. An interdisciplinary challenge]. Unfallchirurg 2011; 114: 688-696

${ }^{25}$ Wahnert D, Lenz M, Schlegel $U$ et al. Cerclage handling for improved fracture treatment. A biomechanical study on the twisting procedure. Acta Chir Orthop Traumatol Cech 2011; 78: 208-214

${ }^{26}$ Wahnert D, Schroder R, Schulze M et al. Biomechanical comparison of two angular stable plate constructions for periprosthetic femur fracture fixation. Int Orthop 2014; 38: 47-53

27 Wirtz DC, Heller KD, Holzwarth U et al. A modular femoral implant for uncemented stem revision in THR. Int Orthop 2000; 24: 134 138

Priv.-Doz. Dr. med. Richard Stange Oberarzt

Dr. med. Moritz Freistühler

Assistenzarzt

Dr. med. Philipp Michel

Assistenzarzt

Dr. med. Clemens Kösters

Oberarzt

Prof. Dr. Michael J. Raschke

Klinikdirektor

Klinik für Unfall-, Hand- und Wiederherstellungschirurgie Universitätsklinikum Münster Waldeyerstraße 1

48149 Münster

richard.stange@ukmuenster.de 